\title{
Theoretical Implications of Triple-Mode RR Lyrae Pulsations
}

\author{
G. Kovács ${ }^{1,2}$, J. R. Buchler ${ }^{1}$ \\ ${ }^{1}$ Department of Physics, University of Florida, Gainesville \\ ${ }^{2}$ Konkoly Observatory, Budapest, Hungary
}

\begin{abstract}
We argue that triple-mode RR Lyrae pulsation with low amplitudes might be quite common. It is shown that until very accurate abundance data become available, triple-mode RR Lyrae stars are the only hope to estimate reliable stellar parameters from the periods alone.
\end{abstract}

\section{Introduction}

The motivation of this study comes from the following observations: (1) most of the radiative RR Lyrae models are linearly excited in some range of temperature in the three lowest order radial modes; (2) many RR Lyrae stars show non-repetitive light variation. Accurate photoelectric observations might reveal some low-amplitude mode contamination (e.g. Fernley et al. 1990); (3) marginally stable modes in a limit cycle may oscillate at low amplitudes because of stochastic mode excitation (Kovács and Buchler, in preparation). Some high-amplitude $\delta$ Scuti stars seem to support the existence of such a multimode pulsation (e.g. Walraven et al. 1992).

We address the following questions in this note: (a) How much the knowledge of the periods of the first three radial modes constrains the derived stellar parameters? (b) Is there any limit cycle in which the other two modes are simultaneously marginally stable?

\section{Determination of $M$ and $Z$}

A large number of RR Lyrae models have been computed in the range $0.6-0.9$, $40-70,6000-8000$ for $M, L$ and $T_{\text {eff }}$ respectively. Two sets of models have been computed for $Z=0.0001$ and $Z=0.001$, both with $X=0.7$ and with solar (i.e. Anders-Grevesse) mixtures. We used the opacities published by Rogers and Iglesias (1992).

The novel sensitivity of the period ratios on $Z$ due to the revised opacities seriously jeopardizes the applicability of the single period ratio method for the mass determination. For three periods, however, the effect of $Z$ can be largely eliminated. The $P_{1} / P_{0}-P_{2} / P_{0}$ versus $P_{0}$ diagram shows very little dependence on $Z$ (Fig. 1). 
In conclusion, if three periods are known, then the $P_{0} \rightarrow P_{1} / P_{0}-P_{2} / P_{0}$ diagram can be used for a mass determination. With this mass, the $P_{0} \rightarrow P_{2} / P_{0}$ diagram constrains the metal abundance.

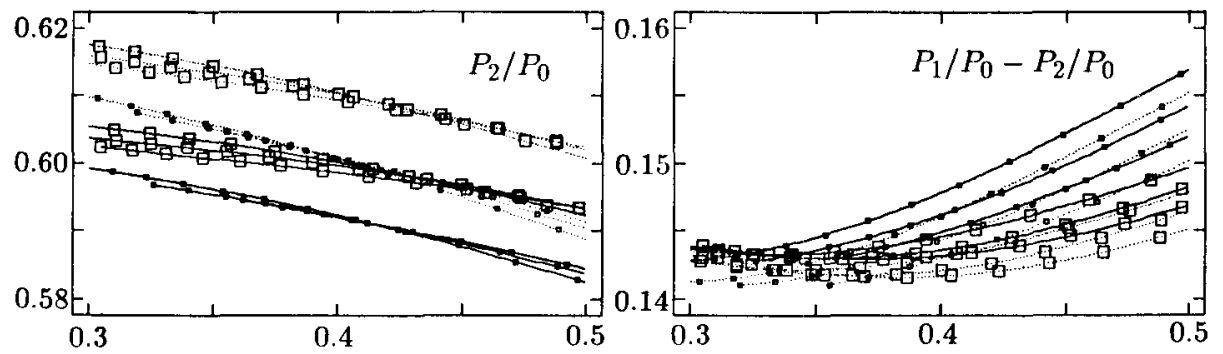

Figure 2. Period ratio diagrams. Dotted lines: $Z=0.0001$, solid lines: $Z=0.001$; small squares: $M=0.6$, large squares: $M=0.8$. At each mass three luminosity values are plotted with $L=40,50,60$. Horizontal axis: $P_{0}$ in [day].

\section{Limit cycle stability}

One possible scenario for triple-mode RR Lyrae pulsation is that the marginally stable modes in a limit cycle get stochastically excited due to turbulent convection. A limit cycle analysis of a sequence of RR Lyrae models showed that simultaneous marginal stability is possible only in the second overtone limit cycle. Predictions of evolution calculations do certainly allow (re)entering into the instability strip from the very hot side, where only the second overtone could be excited.

\section{Conclusions}

Theoretical considerations suggest that triple-mode RR Lyrae pulsation is possible. If it is observed, the periods put further significant constraints on the stellar parameters (especially on $M$ and $Z$ ) over those of the traditional single period ratio method. So far only AC And (Fitch and Szeidl 1976) could be possibly identified as a triple-mode RR Lyrae star pulsating in the first three radial modes. For recent analyses of two RRc stars we refer to Peniche et al. (1989) and Hobart et al. (1991).

Based on the commonly observed non-repetitive variation of the light curves and on the theoretical possibility of triple-mode pulsation, we think it would be very important and profitable to search for low-amplitude pulsations in RR Lyrae stars.

\section{References:}

Fernley, J.A., Skillen, I., Jameson, R.F. and Longmore, A.J. 1990, MNRAS, 242, 685.

Fitch, W.S. and Szeidl, B. 1976, Ap. J., 203, 616.

Hobart, M.A., Peña, J.H. and Peniche, R. 1991, Rev. Mexicana Astr. Ap., 22, 275.

Peniche, R., Gomez, T., Parrao, L. and Peña, J.H. 1989, Astr. Ap., 209, 59.

Rogers, F.J. and Iglesias, C.A. 1992, Ap. J. Suppl., 79, 507.

Walraven, Th, Walraven, J. and Balona, L.A. 1992, MNRAS, 254, 59. 